\title{
General Entomology/Intomologia Geral Diversidade de abelhas (Hymenoptera: Apoidea) e visitação floral em uma área de Mata Atlântica no Sul do Brasil
}

\author{
Alexandre Somavilla ${ }^{\circledR}{ }^{\bowtie}$, Karine Schoeninger¹, David Silva Nogueira1 ${ }^{1}$ \& Andreas Kohler ${ }^{2}$
}

1. Laboratório de Hymenoptera, Coordenação de Biodiversidade, Instituto Nacional de Pesquisas da Amazônia. Manaus, AM, Brasil. 2. Laboratório de Entomologia, Universidade de Santa Cruz do Sul. Santa Cruz do Sul, RS, Brasil.

\section{EntomoBrasilis 11 (3): 191-20o (2018)}

Resumo. A grande riqueza de abelhas está atrelada à grande diversidade de plantas com flores, que possuem certas atratividades para garantir a visita às flores, e consequente polinização. No Rio Grande do Sul, estudos sobre a fauna de abelhas e a flora apícola associada já foram realizados, porém ainda são escassos. Desta forma, correlacionamos os táxons de abelhas e espécies de plantas coletados em uma área florestal urbana e verificamos as relações ecológicas e tróficas entre elas, especialmente ao nicho da abelha exótica Apis mellifera Linnaeus, sobre as abelhas silvestres. Coletamos nas flores um total de 2.772 abelhas determinadas em cinco famílias, 54 gêneros e 88 táxons. Apidae foi a família melhor representada com 35 espécies e 2.047 indivíduos. As abelhas foram coletadas em 43 espécies de plantas classificadas em 19 famílias botânicas sendo Asteraceae a mais visitada. A planta com o maior número de abelhas coletadas foi Citrus sinensis (L.), 30\% do total, tendo A. mellifera a mais representativa. $34 \%$ dos táxons de abelhas estão correlacionados às espécies de plantas visitadas, contudo, quando verificamos esta correlação excluindo a espécie A. mellifera, ocorre um aumento significativo, atingindo 93\%. A manutenção deste fragmento vegetal a suas flores é importante para conservação da biodiversidade apifauna local.

Palavras-chave: Abelhas nativas, Apis mellifera; polinização; sobreposição de nicho trófico; visitantes florais.

\section{Diversity of bees (Hymenoptera: Apoidea) and floral visitation in an Atlantic Forest area in southern Brazil}

\begin{abstract}
The high bees' richness is associated to the great diversity of flowering plants, which have some attractiveness to ensure flowers visiting and the consequent pollination. In Rio Grande do Sul state, studies on the bee fauna associated with bee flora have already been made but are uncommon. In this way, the taxa of bees and plants species visited were correlated, and the ecological and trophic relations between them, particularly in relation to the niche of the exotic bee Apis mellifera Linnaeus on wild bees were analyzed, in an urban forest area. We collected 2,772 bees from the flowers, determined in five families, 54 genera and 88 taxa. Apidae was the best family represented with 35 species and 2,047 individuals. The bees were collected from 43 plants species, classified in 19 botanical families, Asteraceae are the most visited. The plant with the largest number of bees collected was Citrus sinensis (L.), $30 \%$ of the bees, and the A. mellifera the most representative. $34 \%$ of the taxa of bees are correlated to the species of plants visited, however, when we see this correlation excluding A. mellifera, there is a significant increase, reaching $93 \%$. The maintenance of this plant fragment and the flowers is important for preserving the bees' biodiversity in this site.
\end{abstract}

Keywords: Native bees; Apis mellifera; pollination; trophic niche overlap; flower visitors.

IP ela grande diversificação estrutural, comportamental e taxonômica, os Apoidea formam um grupo de grande importância para a polinização de plantas, principalmente as abelhas que, em geral, dependem quase exclusivamente dos recursos obtidos nas flores (RoubiK 1989; Halinski et al. 2015). Estimativas mostram que são conhecidas cerca de 20.500 espécies de abelhas para o mundo (ITIS 2010), sendo que a grande maioria dessas possui hábitos solitários e apenas 1.000 espécies sociais (Imperatriz-FonseCa \& Nunes-Silva 2010).

Essa grande riqueza de abelhas está atrelada à grande diversidade de plantas com flores, que exprimem estreita relação (Milet-Pinheiro \& Schlindwein 2008; Santos et al. 2010), pois as plantas possuem certas atratividades para garantir a visita, e consequente polinização, como modificações estruturais, variações de formas, cores e odores, além da disponibilização de recursos (BАRTH 1991). Além de Apis mellifera Linnaeus, as abelhas nativas do Brasil, tanto sociais quanto solitárias, são fundamentais no processo de polinização (Freitas et al. 2014). As abelhas buscam visitar flores para coletar material para estruturação do ninho como resinas e óleos (ALvEs-Dos-SANTos et al. 2007), para atração sexual, como essências (ELtz et al. 1999) ou para alimentação, como pólen e néctar (Silva et al. 2013).

Levantamentos prévios que estudaram a fauna de abelhas e seus recursos florais utilizados foram realizados em vários biomas do Brasil como na Amazônia (Thomazinı \& Thomazinı 2002), na
Edited by:

William Costa Rodrigues

\section{Article History:}

Received: $11 . v i .2018$

Accepted: 04.ix.2018
Corresponding author:

Alexandre Somavilla

\}alexandre.s@hotmail.com

(3) https://orcid.org/0000-0002-8174-7418
Funding agencies:

$\Delta$ Without funding declared 
Caatinga (SANTos et al. 2010), no Cerrado (ANDEna et al. 2005) e Mata Atlântica (ANTONini \& MARTins 2003).

O Estado do Rio Grande do Sul, apesar do seu tamanho territorial relativamente pequeno, possui uma grande diversidade de ecossistemas (RADAMBRASIL 1981). Até o momento, estudos sobre a fauna de abelhas e a flora apícola associada no estado foram realizados na região da Serra do Sudeste (Schlindwein 1995), no litoral (Alves-Dos-SANTos 1999) e na depressão central, próximo a centro urbano (TRUYLIo \& HARTER-MARques 2007). Cabe ressaltar que estes levantamentos têm sido realizados em ambientes relativamente conservados e estudos em ambientes urbanos praticamente inexistem.

As causas da diminuição e/ou extinção de espécies das abelhas brasileiras são evidentes. O processo contínuo e crescente de urbanização com a ocupação de áreas de vegetação natural para a agricultura ou pecuária são acompanhados pela destruição e devastação dos locais de nidificação e das fontes de alimento (ANDENA et al. 2005). Desta forma, o monitoramento quantitativo das espécies permite verificar a frequência dos indivíduos, identificar as espécies chaves, determinar as preferencias por habitats e/ou recursos florais, bem como o modo de organização das comunidades (ANDENA et al. 2005). Além disso, o monitoramento das espécies favorece a execução de análises que podem revelar que as interações planta-polinizador podem exibir um relativo alto grau de especialização ou, em alguns casos, generalização das visitas (BLÜTHGEN \& KLEIN 2011; AGUIAR et al. 2012).

Com o objetivo de contribuir para o conhecimento de abelhas, no presente estudo analisamos a diversidade de abelhas, bem como correlacionamos estas com as espécies de plantas coletadas em uma Floresta Estacional Decidual de Mata Atlântica imersa em uma matriz urbana no município de Santa Cruz do Sul, Rio Grande do Sul, Brasil.

\section{MATERIAL E MÉTODOS}

Área de estudo. O Cinturão Verde situa-se dentro da área urbana do município de Santa Cruz do Sul, entre as coordenadas $29^{\circ} 43^{\prime} 00 " \mathrm{~S}-52^{\circ} 25^{\prime} \mathrm{OO}$ ” W, na região da Encosta Inferior do Nordeste do estado do Rio Grande do Sul, na unidade geomorfológica correspondente aos patamares da Serra Geral (Collischonn 2001).

A vegetação predominante é a Floresta Estacional Decidual do Bioma Mata Atlântica (IBGE 1986) e o clima, segundo a classificação de Köppen, é do tipo Cfa (Subtropical úmido). Durante o período de coleta, a flutuação da temperatura anual foi de $02{ }^{\circ} \mathrm{C}$ a $34^{\circ} \mathrm{C}$ com $48 \mathrm{~mm}$ a $278 \mathrm{~mm}$ de flutuação da precipitação anual (EstaÇÃo METEOROLÓGiCA DA UNIVERSIDADE DE Santa CRuZ do Sul 2015).

Métodos de coleta e conservação das abelhas. As abelhas foram coletadas através de buscas ativas com redes entomológicas, por 48 meses entre os anos de 2005 a 2008, durante a visitação floral nas plantas em floração; essas coletas aconteceram de maneira aleatória de acordo com o período de floração de cada uma das espécies de plantas, por aproximadamente 1 hora ao dia, em diferentes horários (dandose ênfase ao período de maior incidência de sol, entre $10 \mathrm{~h}$ e 14 h), em cada floração, objetivando associar as espécies de abelhas com as plantas visitadas.

Todas as abelhas coletadas foram depositadas na Coleção Entomológica de Santa Cruz do Sul (CESC) e as identificações foram conforme SiLveira et al. (2002). As espécies vegetais foram coletadas e encaminhadas para o Laboratório de Botânica e depositadas no Herbário da Universidade de Santa Cruz do Sul (HCB), seguindo-se as recomendações usuais.
Análise dos dados. A dominância foi calculada através da fórmula $\mathrm{D}=(\mathrm{i} / \mathrm{t}) \times 100$, onde $\mathrm{D}=$ Dominância $(\%), \mathrm{i}=$ número total de indivíduos de uma determinada espécie e $\mathrm{t}=$ número total de indivíduos coletados, onde $\mathrm{Ed}=>10 \%$ Eudominante, $\mathrm{D}$ $=5-10 \%$ Dominante, $\mathrm{Sd}=2-5 \%$ Subdominante, $\mathrm{Rc}=1-2 \%$ Recessiva e $\mathrm{Rr}=<1 \%$ Rara (PALISSA et al. 1977).

Foi utilizado o inverso do índice de Diversidade de Simpson (SIMPSON 1949) para calcular a largura do nicho de microhabiat (neste caso refere-se aos recursos florais), o qual seguiu a seguinte fórmula:

$$
N B=\frac{1}{\sum^{n}={ }_{\iota} p i 2}
$$

onde $p$ é a proporção da categoria de microhabiat, $i$ e $n$ é o número total de categorias. $\mathrm{O}$ cálculo da largura de nicho a partir do inverso do Índice de Diversidade de Simpson gera valores de 1 a 12,7 onde 1 corresponde ao uso exclusivo de apenas um micro-habitat (especialistas) e 12,7 corresponde ao uso de todos os microhabitats categorizados (generalistas).

A diversidade de abelhas foi estimada através do índice de Shannon-Wiener $\left(\mathrm{H}^{\prime}\right)$ e a equitabilidade $(\mathrm{J})$ através do índice de Pielou (1975). Para as medidas de correlação linear entre as espécies de abelhas e espécies de plantas foi utilizado o coeficiente de correlação de Pearson (r). As análises foram conduzidas no software R, versão 3.1.o (R Development CoRe TEAm 2014), usando o pacote de dados Vegan (Orsanen et al. 2013).

\section{RESULTADOS}

Durante o período de estudo, foram capturadas nas flores um total de 2.772 abelhas determinadas em cinco famílias, 54 gêneros e 88 táxons. Apidae foi a família melhor representada ( 35 espécies em 21 gêneros - 2.047 indivíduos), seguida por Halictidae (13 espécies em 13 gêneros - 452 indivíduos), Megachilidae (28 espécies em 12 gêneros - 190 indivíduos), Andrenidae (cinco espécies em quatro gêneros - 63 indivíduos) e Colletidae (sete espécies em sete gêneros - 22 indivíduos) (Tabela 1).

O índice de Pielou mostrou a dominância de poucas espécies. Para as abelhas sociais, as espécies com maior representatividade são A. mellifera e Tetragonisca angustula (Holmberg), já para as espécies não sociais a maior abundância é encontrada em Augochlora sp. e Augochloropsis sp. O índice de diversidade alcançado neste trabalho foi de $H^{\prime}=2,6$. Em relação a equitabilidade, o índice de Pielou $(\mathrm{J}=0,58)$ indicou haver dominância de uma ou mais espécies de abelhas, sendo que no presente estudo a dominância está atrelada, predominantemente, a $A$. mellifera (1.193 indivíduos), as outras espécies dominantes foram T. angustula (198 indivíduos), Augochloropsis sp. (167 indivíduos), Augochlora sp. (139 indivíduos) e Melissoptila sp. (138 indivíduos).

As abelhas foram coletadas em 43 espécies de plantas determinadas em 19 famílias botânicas (Tabela 2); as famílias com maior número de espécies vegetais foram Asteraceae com 12 espécies, Apiaceae e Fabaceae com cinco espécies cada. A planta com o maior número de abelhas coletadas foi Citrus sinensis (L.) com 845 (30\% do total de abelhas, destas 89,82\% era A. mellifera) seguida por Schinus terebinthifolius Raddi (287 espécimes) e Ocimum selloi Benth. (243 espécimes). As plantas com maior número de espécies de abelhas foram $O$. selloi com 37 espécies, Sida potentilloides A.St.-Hil. (34) e Eryngium sp.1 (30).

A espécie $A$. mellifera, única eudominante, visitou 30 espécies de plantas, sendo uma das mais generalistas do estudo (Tabela 1), contudo a maior representatividade desta espécie foi encontrada em $C$. sinensis (759 indivíduos), o que consequentemente fez 


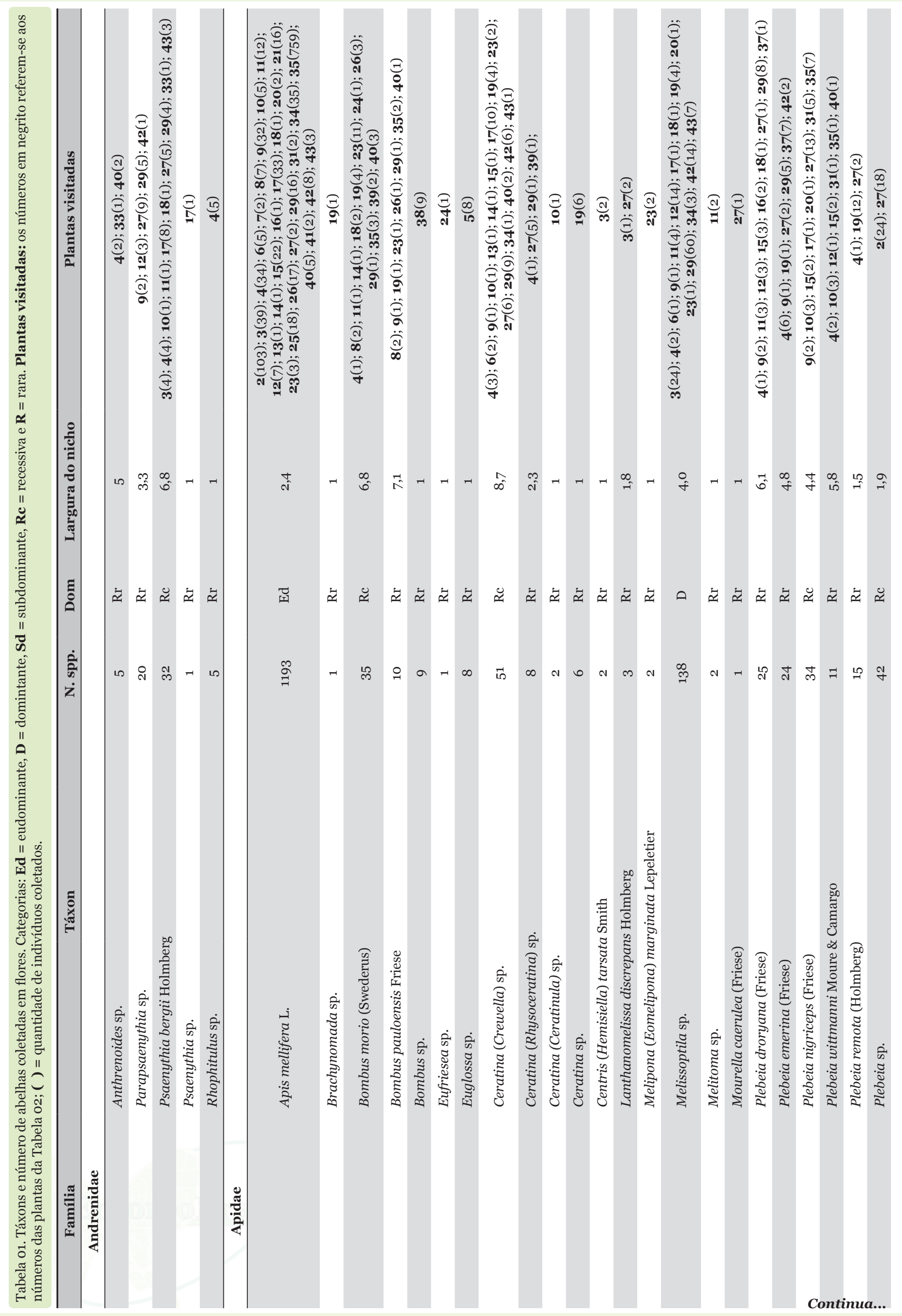




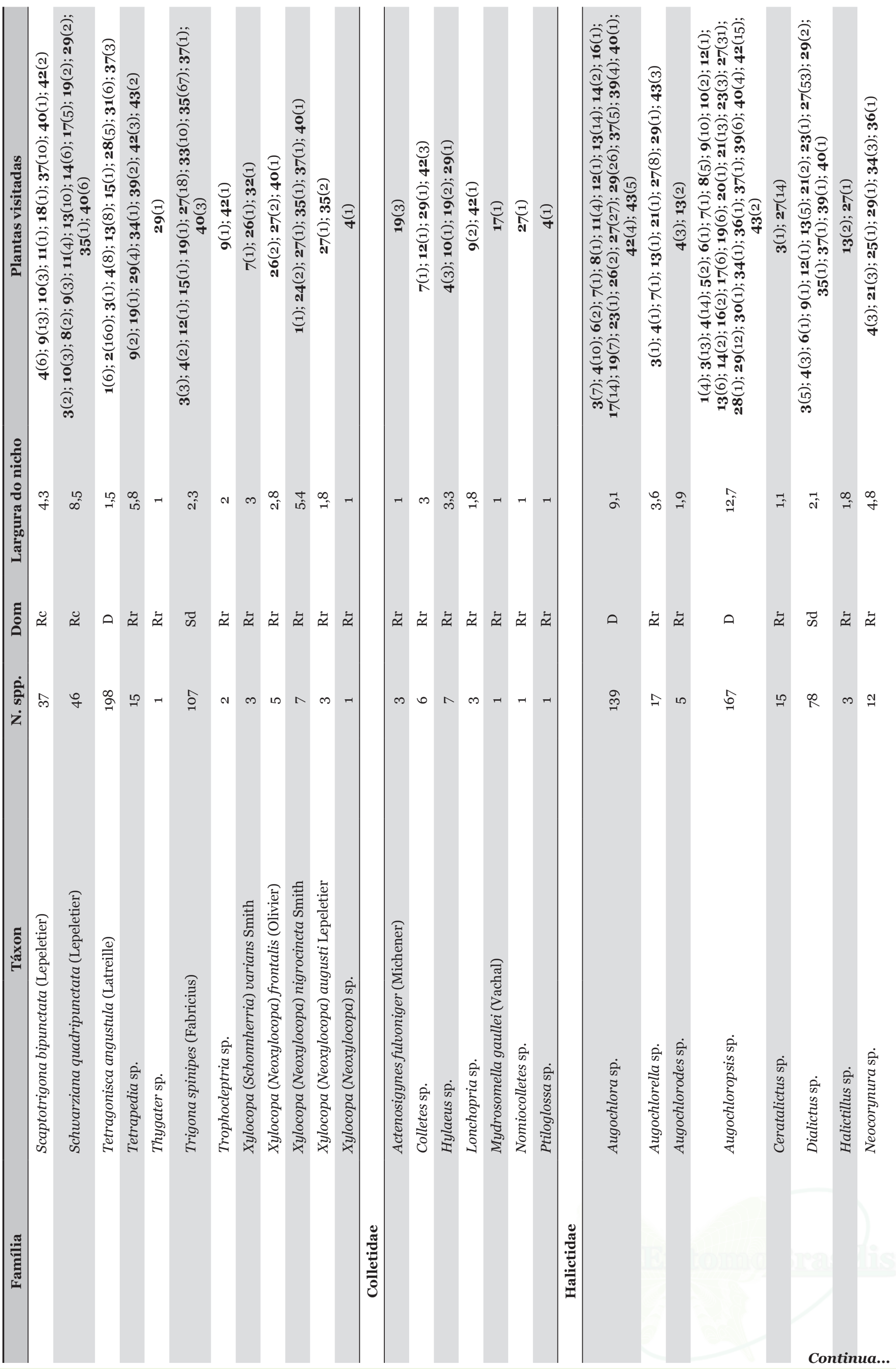




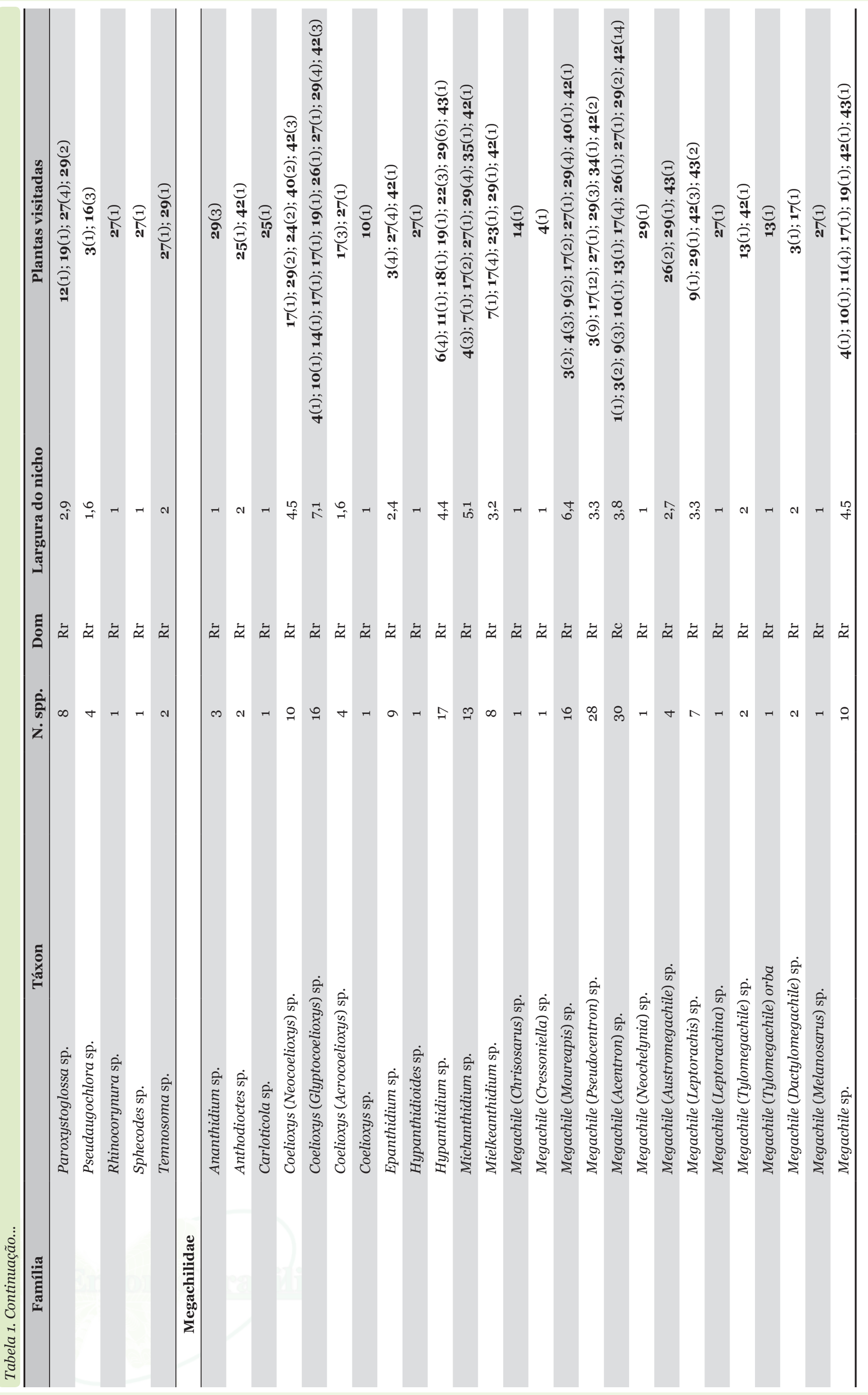


Tabela 2. Espécies de plantas com os respectivos números de abelhas coletadas e espécies de abelhas em cada planta.

\begin{tabular}{llll}
\hline \multicolumn{1}{c}{ Família } & Espécie & N. de Abelhas & N$^{\circ}$ espécies de abelhas/planta \\
\hline Amaranthaceae & &
\end{tabular}
1. Celosia cristata L.
12
4

\section{Anacardiaceae}

2. Schinus terebinthifolius Raddi

287

Apiaceae

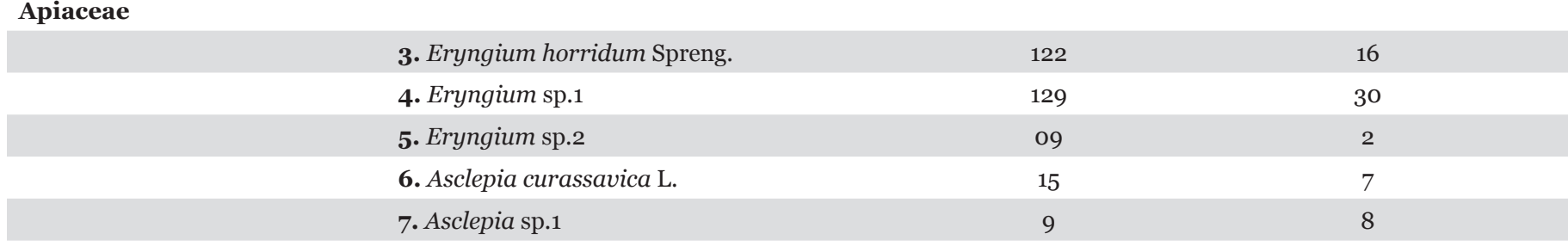

\section{Asteraceae}

Commelinaceae

8. Baccharis phyteumoides DC. $19 \quad 5$

9. Baccharis trimera Less.

10. Baccharis sp.1 33

11. Baccharis $\mathrm{sp} .2$

12. Baccharis sp. $3 \quad 36$

13. Elephantopus mollis Kunth 52

14. Eupatorium purpureum L. 15

15. Eupathorium sp. $\quad 32$

16. Mikania cordifolia Willd 10

17. Senecio brasiliensis Less. $\quad 111$

18. Vernonia oxyodonta Malme 7

19. Vernonia polyanthes Less 60

60

20. Commelina benghalensis L.

Erythroxylaceae

21. Erythroxylum myrsinites Mart.

41

6

Fabaceae

22. Desmodium affine Schltdl

23. Indigofera suffruticosa Mill

24. Inga vera Willd.

25. Lonchocarpus campestres Mart.

26. Mimosa bimucromata DC.

3

3

$26-9$

$4 \quad 3$

$20 \quad 4$

\section{Lamiaceae}

27. Ocimum selloi Benth

243

37

Lauraceae

28. Cinnamomum sp.

6

2

Malvaceae

29. Sida potentilloides $\mathrm{L}$.

196

34

Meliaceae

3o. Trichilia sp.

1

1

\section{Oleaceae}

31. Ligustrum japonicum Thunb

Passifloraceae

32. Passiflora edulis Sims.

1

1

Poaceae

33. Zea mays L.

Rubiacae

34. Uncaria tomentosa DC.

46

8

Rutaceae

35. Citrus sinensis L.

845

8

Sapindaceae 
Tabela 2. Continuação...

\begin{tabular}{llcc}
\hline \multicolumn{1}{c}{ Família } & \multicolumn{1}{c}{ Espécie } & N. de Abelhas & No espécies de abelhas/ planta \\
\hline & 36. Cupania vernalis Cambess & 2 & 2 \\
\hline Solanaceae & 37. Allophylus edulis Radlk. & 30 & 8 \\
& 38. Solanum acerosum Sendtn & 9 & 1 \\
& 39. Solanum variable L. & 16 & 6 \\
\hline 40. Solanum sp. & 34 & 15 \\
\hline Verbenaceae & 41. Cestrum nocturnum L. & 2 & 1 \\
\hline & 42. Lantana sp. & 93 & 19 \\
\hline
\end{tabular}

com que seu nicho fosse pouco representativo (nicho alimentar $=2,4$ ) em comparação com as demais espécies.

As espécies dominantes $T$. angustula visitou 11 espécies de plantas e Melissoptila sp.1 (15), com nicho $\mathrm{n}=1,5$ e $\mathrm{n}=4,0$, respectivamente, uma vez que estas duas espécies foram mais abundantes em uma única espécie vegetal (Tabela 1). Porém, para as espécies Augochloropsis sp.1 (29 espécies vegetais) e Augochlora sp.1 (21 espécies vegetais), houve uma distribuição homogênea entre os indivíduos e respectivos recursos.

De acordo com as análises, 34\% (p = 0,02) dos táxons de abelhas são correlacionados às espécies de plantas visitadas (Figura 1A).
Contudo, quando verificamos está correlação excluindo a espécie exótica $A$. mellifera que é eudominante, ocorre um aumento significativo na correlação, passando a $93 \%\left(\mathrm{p}=2,2^{*} 10^{-16}\right)$ (Figura 1B).

Em relação a distribuição das abelhas sociais e não sociais ao longo de 48 meses de coletas, observa-se uma predominância das abelhas sociais, exceto para o mês abril (Figura 2). Também se verifica a ausência de abelhas não-sociais durante os meses junho e julho que foram os meses de temperaturas mais amenas no Rio Grande do Sul.
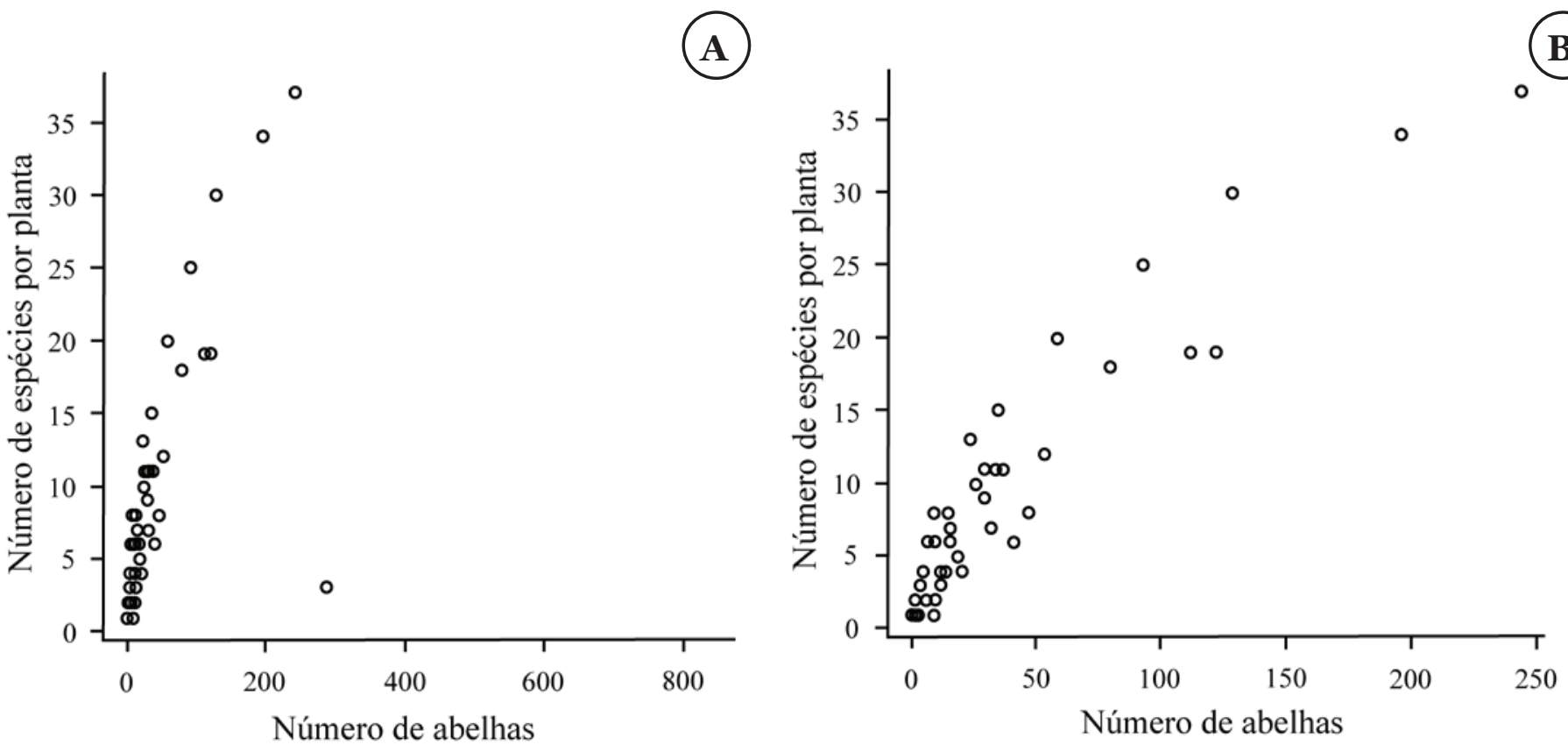

Figura 1. Análise de correlação de Pearson entre os táxons de abelhas com as espécies de plantas; (A) utilizando Apis mellifera; (B) análise sem Apis mellifera.

\section{DISCUSSÃO}

Os Apidae corbiculados tendem a aumentar sua diversidade em direção à linha do Equador, sendo mais bem representados em florestas tropicais quentes e úmidas; Meliponini são abelhas mais diversificadas em florestas úmidas (Roubik 1989). A maior abundância de indivíduos foi encontrada em Apini e Meliponini, devido ao comportamento eussocial dessas abelhas, à perenidade das colônias e aos hábitos generalizados de forrageio (RoubiK 1989; Michener 2007). E apesar de Meliponini ser uma das tribos com maior riqueza de espécies neste estudo, em comparação com outros, é considerada com índices baixos (ANDENA et al. 2005), esta pode estar relacionada ao atual estado de degradação da vegetação no estado do Rio Grande do Sul, que causa a escassez de sítios de nidificação, uma vez que a maioria dessas abelhas constrói seus ninhos em troncos, e escassez de recursos florais, principalmente de árvores com floração em massa (RouBIK 1989). ZANELLA (2003) vinculou o baixo número de abelhas "sem ferrão" à ação humana, seja pela destruição de ninhos para obtenção de mel ou pela redução da cobertura vegetal, já que elas são sensíveis às alterações ambientes.

Destacamos que as abelhas sociais nativas competem em muitos habitats com a introduzida abelha africanizada, frequentemente causando um efeito negativo na comunidade (PEDRo \& CAMARGo 1991; Wilms et al. 1996; Aguiar \& Martins 2003). Neste estudo, o baixo índice de diversidade $\left(H^{\prime}=2,6\right)$ encontrado é decorrente do grande número de espécies com poucos indivíduos e de poucas espécies com muitos indivíduos, tais como A. mellifera. Porém, alguns autores excluíram esta espécie de suas amostras por considerá-la uma espécie introduzida (Silveira \& CAMPos 1995), o que dificulta a avaliação da apifauna local em comparação com 


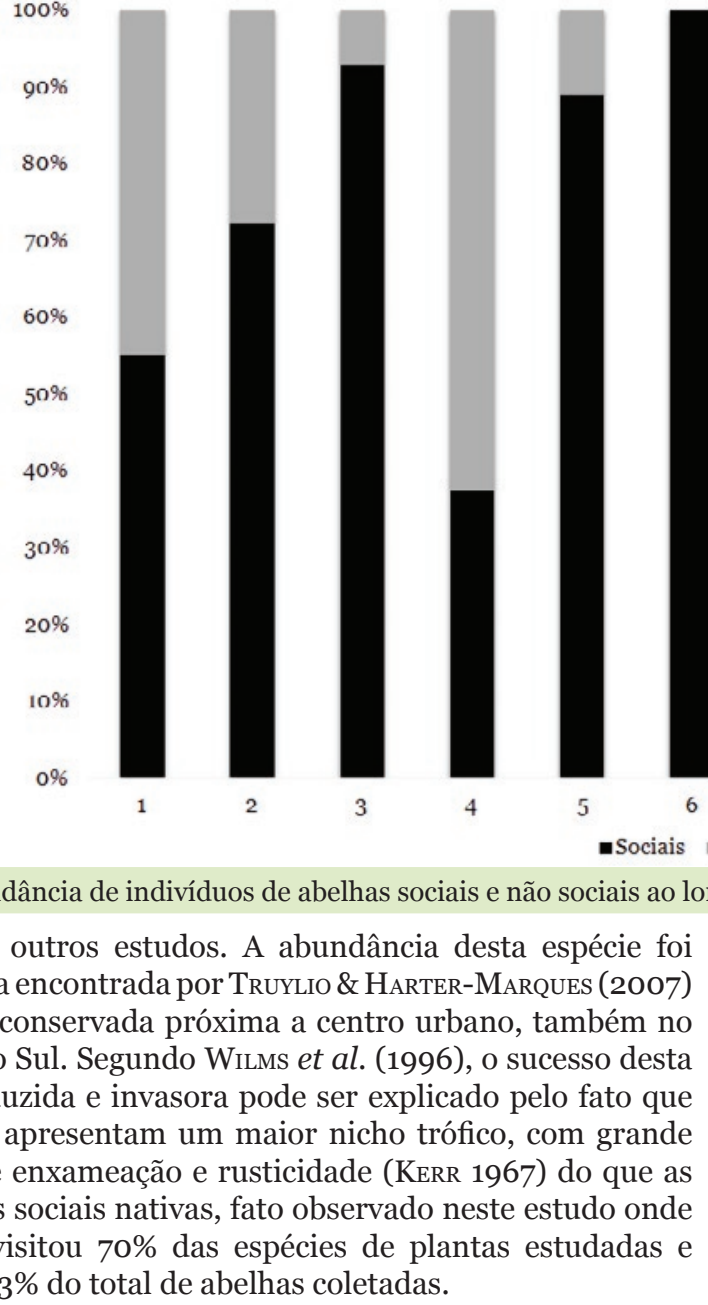
representou $43 \%$ do total de abelhas coletadas.

Quando A. mellifera está presente nos ambientes, a competitividade por recursos (principalmente pólen) e locais para nidificação (Roubik et al. 1986; Pedro \& CAMARgo 1991; WiLms et al. 1996) aumenta, isso reduz os números de espécimes coletados da fauna nativa, além de que, essa espécie é altamente generalista e possui colônias muito numerosas (WINSTON 1987). Essa competição foi estudada por diversos autores: RouBıK et al. (1986) monitoraram a coleta de pólen e néctar por 12 espécies de Meliponini e A. mellifera no Panamá e verificaram que apesar da abelha africanizada compartilhar muitas flores e possuir capacidade de forrageio até 100 vezes maior que as nativas, aparentemente isso não reflete em uma sobreposição. PEDRo \& CAMARGO (1991) concluíram queas plantas visitadas por A.mellifera diferem consideravelmente daquelas visitadas por abelhas solitárias e especializadas, e mesmo com os meliponíneos, cujos atributos comportamentais e morfológicos são mais similares a A. mellifera, a sobreposição das espécies vegetais é baixa. XIA et al. (2007) sugerem que a presença da Apis nos ambientes, pode reduzir as perdas causadas pela ausência de outras abelhas polinizadoras, mas já Gouson \& SPARROw (2008) indicam fortes evidências que sugerem que a sua presença prejudica a fauna de polinizadores nativos afetando suas interações. Essa discussão mostra como é difícil ter um consenso sobre as consequências da presença desta espécie nos ambientes naturais.

Outras espécies frequentes neste estudo foram $T$. angustula e Trigona spinipes (Fabricius), ambas possuem colônias numerosas, e são generalistas, bem adaptadas a diversos ambientes, com uma ampla distribuição e capacidade de dispersão (JAFFé et al. 2014). T. angustula é muito utilizada por criadores para produção de mel, e juntamente com $T$. spinipes, podem ser consideradas como polinizadoras de resgate, já que possuem boa adaptação a ambientes antropizados (GIANNini et al. 2015). Ambas também foram frequentes em outros estudos de

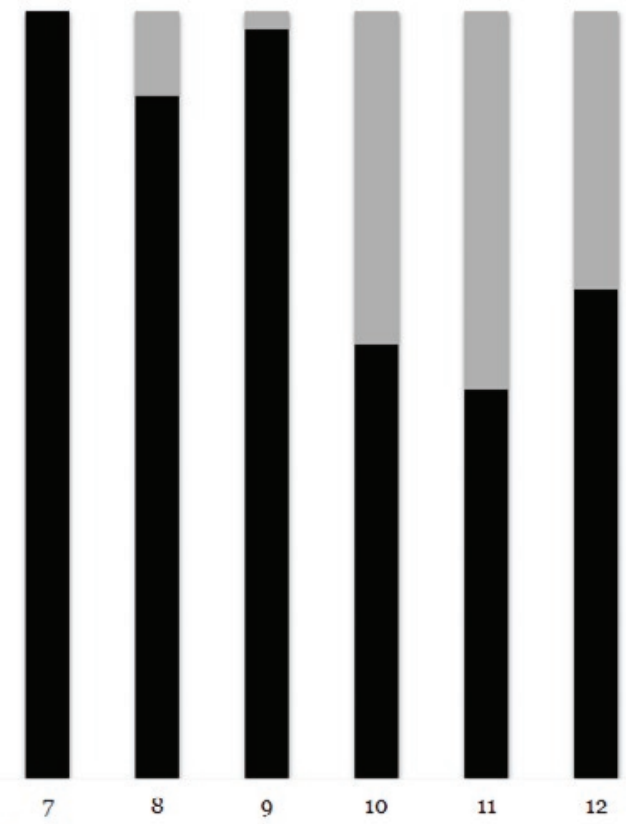

Não sociais abelhas que visitam flores, principalmente em áreas cultivadas de Citrus (Nascimento et al. 2011).

Halictidae é a segunda família em abundância, o que confere com os dados compilados de Pacheco Filho et al. (2015), porém, a riqueza de espécies desta família na área de estudo é baixa quando comparada a outros estudos realizados no Rio Grande do Sul em áreas de parques urbanos preservadas (WitTMann \& HoffmanN 1990; Alves-Dos-Santos 1999; Truylio \& Harter-Marques 2007).

Apesar de Megachilidae ser numerosa no Rio Grande do Sul (Alves-Dos-Santos 1999), essa família mostrou índices intermediários de abundância e riqueza (188 indivíduos e 28 espécies), valores superiores a TRUyLio \& HARTER-MARQUES (2007). Rоuвiк (1989) sugere que o menor número de espécies de Megachilidae e Halictidae pode estar relacionado ao fato de, em sua maioria, apresentarem comportamento de abelhas solitárias (com populações menos numerosas do que as de espécies sociais). Desta maneira, estas abelhas são mais raras e sazonais, dificultando a sua coleta nas flores.

Andrenidae e Colletidae são mais diversificadas nas regiões temperadas e subtropicais do sul da América do Sul (Roubik 1989; Silveira et al. 2002) e, quando não ausentes, são pouco presentes nas comunidades estudadas na maior parte do Brasil. Neste estudo foram 63 espécimes de Andrenidae e 22 de Colletidae números próximos aos coletados por Truylio \& HARTER-MARQUES (2007), porém naquela abordagem as abelhas não foram associadas às plantas.

As abelhas sociais foram ativas no processo de visitação floral durante todos os meses do ano, não sendo encontrado um padrão sazonal, diferentemente das abelhas solitárias que tem sua atividade reduzida nos meses de temperaturas mais baixas, geralmente entre junho e agosto. A ausência deste padrão, mostrada principalmente em Apini e Meliponini, tem sido frequentemente explicada em decorrência do próprio comportamento eussocial das mesmas, com construção de ninhos perenes. Além disso, a discrepância entre a abundância de abelhas de acordo com o seu comportamento nos meses de inverno e com temperaturas mais baixas (junho-setembro), quando coletamos predominantemente abelhas sociais, também se deve à manutenção dos ninhos destas abelhas, neste período (ANDEna et al. 2005).

Os fragmentos florestais e a vegetação adjacente a estes fragmentos são importantes para a manutenção e sobrevivência das abelhas 
no estado do Rio Grande do Sul. Nosso estudo demonstra que indivíduos isolados de árvores ou arbustos nativos apresentaram um papel fundamental para a manutenção da apifauna local. As espécies de Eryngium e Baccharis, por exemplo, garantem recursos indispensáveis para 48 e 58 espécies de abelhas, respectivamente. $O$. selloi e $S$. potentilloides garantiram recursos para 37 e 34 espécies de abelhas, respectivamente.

A grande quantidade de flores pequenas e rasas, arranjadas em inflorescências nas espécies vegetais acima citadas, facilita o acesso para as abelhas. $\mathrm{O}$ alto número de asteráceas visitadas também pode ser explicado pela grande quantidade de inflorescências por indivíduo e pelo fácil acesso morfológico das abelhas aos recursos florais (CERANA 2004), como nas espécies Baccharis. Com exceção das flores de $S$. potentilloides, que são pequenas, arranjadas de maneira individual, mas que fornecem pólen e néctar para os visitantes. Vários levantamentos apifaunísticos em diferentes locais do Brasil mostraram a importância desta família como fornecedora de recursos florais (Alves-Dos-SANTos 1999; LOCATELLI \& MACHADO 2001). Fabaceae é frequentemente visitada por abelhas de porte robusto, que retiram o pólen por meio de vibração da musculatura de vôo (BuchmanN 1985). Nas regiões tropicais, entre as plantas mais comuns, que apresentam anteras poricidas, destacam-se as Fabaceae e Solanaceae (BuchmanN 1985).

Além de utilizarem plantas nativas, muitas abelhas também visitaram flores de plantas frutíferas cultivadas, como as de C. sinensis e de plantas ornamentais de outras regiões, como Verbena sp., ambas foram visitadas por 11 espécies de abelhas. A presença desses visitantes é de fundamental importância para garantir uma maior produtividade vegetal. Nesse caso, a ausência de recursos florais nativos pode, pelo menos em parte, ser compensada por espécies cultivadas selecionadas. O manejo adequado e ecologicamente sustentável de cultivares, plantas ornamentais e da vegetação do entorno é, nesse contexto, uma alternativa para garantir alta produtividade dos cultivares e ao mesmo tempo a manutenção da apifauna nativa (MILET-PINHeiro \& SCHLINDWEIN 2008; Freitas et al. 2014).

A espécie A. mellifera pode competir por recursos com as abelhas sociais nativas, principalmente Meliponini, causando um efeito negativo na comunidade, devido a grande abundância e, geralmente, causando uma baixa diversidade local. Apesar do Cinturão Verde de Santa Cruz do Sul ser um pequeno fragmento, no entorno de área urbana e agrícola, o mesmo abriga uma comunidade de abelhas composta por um grande número de espécies, embora menos rica que outras áreas da Mata Atlântica, porém a manutenção desse fragmento é importante para a conservação da biodiversidade da apifauna local, principalmente porque mantém os sítios de nidificação e as fontes de recursos para essas abelhas nativas.

\section{REFERÊNCIAS}

Aguiar, C.M.L. \& C.E. Martins, 2003. The bee diversity of the Tabuleiro vegetation in the Guaribas Biological Reserve (Mamanguape, Paraíba, Brazil), p. 209-216. In: Melo, G.A.R. \& I. Alves-Dos-Santos (Eds.). Apoidea Neotropica: homenagem aos 90 anos de Jesus Santiago Moure. Criciúma, UNESC.

Aguiar, C.M.L., G.M.M. Santos, C.F. Martins \& S.J. Presley, 2012. Trophic niche breadth and niche overlap in a guild of flowervisiting bees in a Brazilian dry forest. Apidologie: 44: 153-162. DOI: https://doi.org/10.1007/s13592-012-0167-4.

Alves-Dos-Santos, I., 1999. Abelhas e plantas melíferas da Mata Atlântica, restinga e dunas de Litoral norte do Estado do Rio Grande do Sul. Revista Brasileira de Entomologia, 43: 191223.

Alves-Dos-Santos, I., I.C. Machado \& M.C. Glanglianone, 2007. História Natural das Abelhas Coletoras de Óleo. Oecologia Brasiliensis, 11: 544-557.
Andena, S.R, L.R. Bego \& M.R. Mechi, 2005. A Comunidade de abelhas (Hymenoptera, Apoidea) de uma área de cerrado (Corumbataí, SP) e suas visitas às flores. Revista Brasileira de Zoociências, 7: 55-91.

Antonini, Y. \& R.P. Martins, 2003. The value of a tree species (Caryocar brasiliense) for a stingless bee Melipona quadrifasciata quadrifasciata. Journal of Insect Conservation, 7: 167-174.

Barth, F.G., 1991. Insects and flowers - the biology of a partnership. Princeton University Press, Princeton. 424 p.

Blüthgen, N. \& A.M. Klein, 2011. Functional complementarity and specialisation: the role of biodiversity in plant-pollinator interactions. Basic and Applied Ecology, 12: 282-291. DOI: https://doi.org/10.1016/j.baae.2010.11.001.

Buchmann, S.L., 1985. Bees use vibration to aid pollen collection from nonporicidal flowers. Journal of the Kansas Entomological Society, 58: 517-525.

Cerana, M.M., 2004. Flower morphology and pollination in Mikania (Asteraceae). Flora Morphology, Distribution, Functional Ecology of Plants, 199: 168-177. DOI: https://doi.org/10.1078/0367-2530-00145

Collischonn, E. 2001. O Espaço Natural na Região do Vale do Rio Pardo - Algumas Considerações, p. 19-46. In: Vogt, O.P. \& R.L.L. Silveira (Eds.) Vale do Rio Pardo: (re)conhecendo a região. Santa Cruz do Sul, Editora UNISC.

Dec, E \& Mouga, D.M.D.S. 2014. Diversidade de abelhas (Hymenoptera: Apidae) em área de Mata Atlântica em Joinville, Santa Catarina. Acta Biológica Catarinense, 1: 1527. DOI: https://doi.org/10.21726/abc.v1i2.91.

Eltz, T., W.M. Whitten, D.W. Roubik \& K.E. Linsenmair, 1999. Fragrance Collection, Storage, and Accumulation by Individual Male Orchid Bees. Journal of Chemical Ecology, 25: 157-176.

Estação Meteorológica da Universidade de Santa Cruz do Sul, 2015. Disponível em: <http://online.unisc.br/tempo/home. seam $>$. Acesso em: 30.i.2016.

Freitas, B.M., A.J.S. Pacheco Filho, P.B. Andrade, C.Q. Lemos, E.E.M. Rocha, N.O. Pereira, A.D.M. Bezerra, D.S. Nogueira, R.L. Alencar, R.F. Rocha \& K.S. Mendonça, 2014. Forest remnants enhance wild pollinator visits to cashew flowers and mitigate pollination deficit in NE Brazil. Journal of Pollination Ecology, 12: 22-30.

Giannini T.C., L.G. Garibaldi, A.L. Acosta, J.S. Silva, K.P. Maia, A.M. Saraiva, P.R. Guimarães, \& A.M.P. Kleinert, 2015. Native and nonnative supergeneralist bee species have different effects on plantbee networks. PLoS One, 10: 1-13. DOI: https://doi.org/10.1371/journal.pone.0137198.

Goulson, D. \& K.R. Sparrow, 2008. Evidence for competition between honeybees and bumblebees; effects on bumblebee worker size. Journal of Insect Conservation, 13: 177-181. DOI: https://doi.org/10.1007/s10841-008-9140-y.

Halinski, R., A.L. Dorneles \& B. Blochtein, 2015. Bee assemblage in habitats associated with Brassica napus L. Revista Brasileira de Entomologia, 59: 222-228. DOI: https://doi.org/10.1016/j.rbe.2015.07.001.

Imperatriz-Fonseca, V. \& P. Nunes-Silva, 2010. As abelhas, os serviços ecossistêmicos e o Código Florestal Brasileiro. Biota Neotropica, 10: 1-4. DOI: https://doi.org/10.1590/s167606032010000400008 .

Instituto Brasileiro de Geografia e Estatística (IBGE). 1986. Projeto RADAMBRASIL: Levantamento de Recursos Naturais. Rio de Janeiro, IBGE. 791p.

ITIS (Interagency Taxonomic Information System), 2010. Integrated Taxonomic Information System. World Bee ChecklistProject.Disponívelem: $<$ http://www.catalogueoflife. org/annual-checklist/2010/details/database/id/67>. Acesso em: 30.i.2016.

Jaffé, R., F.C. Pioker-Hara, C.F. Santos, L.R. Santiago, D.A. Alves, A. Kleinert T.M. Francoy, M.C. Arias \& V.L. Imperatriz-Fonseca, 2014. Monogamy in large bee societies: 
a stingless paradox. Naturwissenschaften, 101: 261-264. DOI: https://doi.org/10.1007/s00114-014-1149-3.

Kerr, W.E., 1967. The history of the introduction of African bees to Brazil. South African Bee Journal, Petroria, África do Sul, 39: $3-5$.

Locatelli, E. \& I.C.S. Machado, 2001. Bee diversity and their floral resources in a fragment of a tropical altitudinal wet Forest ("Brejos de Altitude") in Northeastern Brazil. Acta Horticulturae, 561: 317-325. DOI: https://doi.org/10.1766o/ actahortic.2001.561.48.

Michener, C.D., 2007. The Bees of the World. The Johns Hopkins University Press. 2nd Ed. 953 p.

Milet-Pinheiro, P. \& C. Schlindwein, 2008. Comunidade de abelhas (Hymenoptera, Apoidea) e plantas em uma área do Agreste pernambucano, Brasil. Revista Brasileira de Entomologia, 52: 625-636. DOI: https://doi.org/10.159o/ s0085-56262008000400014.

Nascimento, E.T., R. Perez-Maluf, R.A. Guimarães, \& M.A. Castellani, 2011. Diversidade de abelhas visitantes das flores de Citrus em pomares de laranjeira e tangerineira. Revista Brasileira Fruticultura, 33: 111-117. DOI: https://doi.org/10.1590/s0100-29452011005000048.

Oksanen, J., F.G. Blanchet, R. Kindt, P. Legendre, P.R. Minchin, R.B. O'hara, G.L. Simpson, P. Solymos, M.H.H. Stevens \& H. Wagner, 2013. Vegan: Community Ecology Package. R package version 2.0-10.

Pacheco Filho, A.J.S., C.F. Verola, L.W. Lima Verde \& B.M. Freitas, 2015. Bee-flower association in the Neotropics: implications to bee conservation and plant pollination. Apidologie, 46: 530-541. DOI: https://doi.org/10.1007/s13592-014-0344-8.

Palissa, A.E., M. Wiedenroth \& K. Klimt, 1977. Anleitung zum ökologischen Geländepraktikum. Wissenschaftliches Zentrum der Pädagogischen Hochschule Potsdam. 186 p.

Pedro, S.R.M. \& J.M.F. Camargo, 1991. Interactions on floral resources between the Africanized honeybee Apis mellifera $\mathrm{L}$ and the native community (Hymenoptera:Apoidea) in a natural "cerrado" ecosystem in southern Brazil. Apidologie, 22: 397-415. DOI: https://doi.org/10.1051/apido:19910405.

Pielou, E.C., 1975. Ecological Diversity. New York: Wiley. [A general book on ecological diversity. Entropy-based diversity measures. Models (for the distribution of species)].

R Development Core Team, 2014. R: A language and environment for statistical computing. R Foundation for Statistical Computing, Vienna, Austria, ISBN 3-900051-07-0. Disponível em: <http://www.R-project.org/>. Acesso em: 16.xii.2015.

Radambrasil, 1981. Levantamento de Recursos Naturais. Rio de Janeiro, v. 19, Folha SD 22, Projeto Radambrasil. 624 p.

Roubik, D.W. 1989. Ecology and natural history of tropical bees. New York, Cambridge University Press. 514 p.

Roubik, D.W., J.E. Moreno, C. Vergara \& D. Wittmann, 1986. Sporadic food competition with the African honey bee: projected impact on Neotropical social bees. Journal of Tropical Ecology, 2: 97-111. DOI: https://doi.org/10.1017/ s0266467400000699.

Santos, G.M.M., C.M.L. Aguiar, M.A.R. Mello, 2010. Flowervisiting guild associated with the Caatinga flora: trophic interaction networks formed by social bees and social

\section{Suggestion citation:}

Somavilla, A., K. Schoeninger, D.S. Nogueira \& A. Kohler, 2018. Diversidade de abelhas (Hymenoptera: Apoidea) e visitação floral em uma área de Mata Atlântica no Sul do Brasil. EntomoBrasilis, 11 (3): 191-200.

Available on: doi:10.12741/ebrasilis.v11i3.800 wasps with plants. Apidologie, 41: 466-475. DOI: https://doi.org/10.1051/apido/2009081.

Schlindwein, C., 1995. Wildbienen und ihre Trachtpflanzen in einer südbrasilianischen Buschlandschaft: Fallstudie Guaritas, Bestäubung bei Kakteen und Loasaceen. Stuttgart, Ulrich Grauer. 148 p.

Silva, D.P., J. Moisan-De-Serres, D.C. Souza, S.B. HilgertMoreira, M.Z. Fernandes, P.G. Kevan \& B.M. Freitas, 2013. Efficiency in pollen foraging by honey bees: time, motion, and pollen depletion on flowers of Sisyrinchium palmifolium (Asparagales: Iridaceae). Journal of Pollination Ecology, 11: 27-32.

Silveira, F.A. \& M.J.O. Campos, 1995. A melissofauna de Corumbataí (SP) e Paraopeba (MG) e uma análise da biogeografia das abelhas do cerrado brasileiro (Hymenoptera, Apoidea). Revista Brasileira de Entomologia, 39: 371-401.

Silveira, F.A., G.A.R. Melo \& E.A.B. Almeida, 2002. Abelhas Brasileiras: Sistemática e Identificação. Belo Horizonte, $1^{\mathrm{a}}$ Edição. 253p.

Simpson, E.H. 1949. Measurement of diversity. Nature, 163: 688-688.

Thomazini, M.J. \& A.P.B.W. Thomazini, 2002. Diversidade de Abelhas (Hymenoptera: Apoidea) em Inflorescências de Piper hispidinervum (C.DC.). Neotropical Entomology, 31: 27-34. DOI: https://doi.org/10.1590/s1519566x200200010Do004.

Truylio, B. \& B. Harter-Marques, 2007. A comunidade de abelhas (Hymenoptera, Apoidea) em áreas florestais do Parque Estadual de Itapuã (Viamão, RS): diversidade, abundância relativa e atividade sazonal. Iheringia, Série Zoologia, 97: 392-399. DOI: https://doi.org/10.1590/s007347212007000400006 .

Wilms, W., V.L. Imperatriz-Fonseca \& W. Engels. 1996. Resource partioning between highly eusocial bees and possible impact of the introduced Africanized honey bee on native stingless bees in the brasilian atlantic rainforest. Studies on Neotropical Fauna and Environment, 31: 137-151. DOI: https://doi.org/10.1076/snfe.31.3.137.13336.

Winston, M.L. 1987. The biology of the honeybee. Cambridge: Harvard University Press. $281 \mathrm{p}$.

Wittmann, D. \& M. Hoffmann, 1990. Bees of Rio Grande do Sul, southern Brazil (Insecta, Hymenoptera, Apoidea). Iheringia, Série Zoologia, 70: 17-43.

Xia, J., S.G. Sun \& Y.H. Guo, 2007. Honeybees enhance reproduction without affecting the outcrossing rate in endemic Pedicularis densispica (Orobanchaceae). Plant Biology, 9: 713-719. DOI: https://doi.org/10.1055/s-2007-965259.

Zanella, F.C.V. 2003. Abelhas da Estação Ecológica do Seridó (Serra Negra do Norte, RN): aportes ao conhecimento da diversidade, abundância e distribuição espacial das espécies da caatinga, p. 231-240. In: Melo, G.A.R. \& I. Alves-dosSantos (Eds.). Apoidea Neotropica: Homenagem aos 90 anos de Jesus Santiago Moure. Criciúma, Editora UNESC. 320p.

$* * * * * * * * * *$
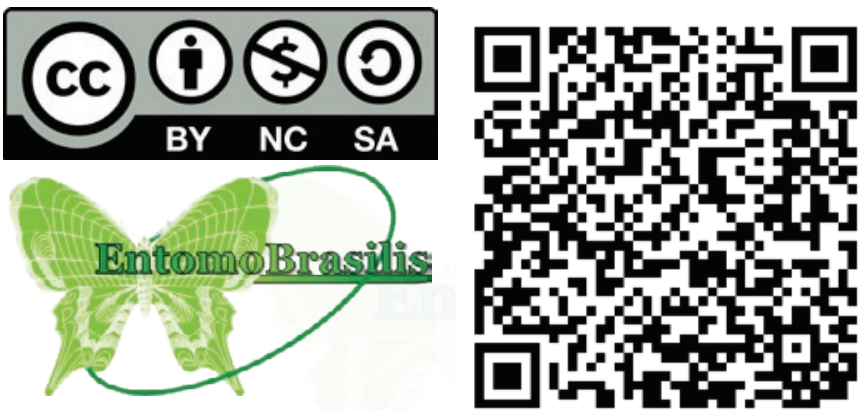